\title{
The Astrophysical Jets (again): as the most complicated inorganic machines known to us
}

\author{
Wolfgang Kundt* \\ Argelander Institut of Bonn University, Germany \\ E-mail: wkundteastro.uni-bonn.de
}

In this talk (at Mondello), I attempt to sketch again my understanding of the universal working scheme of all the astrophysical jet sources, or 'bipolar flows', on both stellar and galactic scales, also called 'microquasars', and 'quasars'. A crucial building block will be their medium (whenever bare): extremely relativistic $\mathrm{e}^{ \pm}$-pair plasma performing quasi loss-free $\mathbf{E} \times \mathbf{B}$-drifts through self-rammed channels, whose guiding equi-partition $\mathbf{E}$ - and $\mathbf{B}$-fields convect the electric potential necessary for eventual single-step post-acceleration, at their 'knots' and terminating 'hotspots', or 'heads'. These electromagnetic fields convect half of the jet's power. The indispensible pair plasma is generated in magnetospheric reconnections of the heavy central rotator. For this reason alone, black holes cannot possibly serve as jet engines.

During its passage from subsonic to supersonic propagation, still inside its deLaval nozzle, the escaping relativistic pair-plasma passes from a relativistic Maxwellian distribution (almost) to that of a (mono-energetic) Deltafunction, of (uniform) Lorentz-factor $\gamma=10^{2 \pm 2}$. Clearly, this transition in velocity distribution - in transit through the deLaval nozzle - is not loss-free; it turns the jet engine into a transient powerful $\gamma$-ray emitter, with photon frequencies reaching up to $\lesssim 10^{26} \mathrm{~Hz}$, (corresponding to electron Lorentz factors $\gamma \lesssim 10^{6}$ ), see page 120 of [Kundt \& Krishna, 2004].

So far, until [Kundt, 2015], all the jets were treated as though propagating in vacuum, as "bare jets". New in 2015 was an allowance for an embedding medium of non-negligible density, most notably encountered in SS 433 (with its fast-moving X-ray and optical spectral lines), but likewise in our Galactic twin jet. Such an embedding medium, gas or plasma, will try to penetrate into the jet channels, but will instead be ionised, expelled, and dragged along by the streaming, extremely relativistic pair plasma, in the form of subrelativistically comoving channel-wall material. In this way, bare jets are converted into (line- and continuum-) emitting "dressed jets". Unfortunately, in 2015, I had been a bit rush: The generalisation from 'bare' to 'dressed' is not exactly straightforward, and should respect stability constraints, in order to (again) be unique; which I hope to present below, in this my second approach.

XII Multifrequency Behaviour of High Energy Cosmic Sources Workshop

12-17 June, 2017

Palermo, Italy

${ }^{*}$ Speaker. 


\section{How to make jets?}

Astrophysical jet sources are among the most delicate engines of the Universe: The engines can eject charges at Very-High Energies (VHE), continually at almost luminal speeds, to astronomical distances of order $\lesssim \mathrm{Mpc}$, for $\gtrsim 10^{7} \mathrm{yr}$ nonstop, in two antipodal directions, in a beamed manner, aimed within an angle of $\gtrsim 1 \%$, whereby all particles arrive with $\gtrsim \mathrm{GeV}$ energies at their termination sites. On arrival, this relativistic $\mathrm{e}^{ \pm}$- pairplasma is converted into a powerlaw-distribution with $\mathrm{e}^{ \pm}$- energies $\lesssim \mathrm{PeV}$. Militaries might appreciate availing of guns of this kind. How does non-animated matter achieve such a difficult task, time and again, on various size and mass scales? After years of intense deliberation, it is my distinct understanding that all the jet engines follow one and the same universal working pattern.

Note that for its multiple tasks, biology uses reliable molecular engines with stable working patterns that can be easily reproduced by both plants and animals; like cells, hearts, lungs, intestines, muscles, nerves, a large number of senses, and all that. But in astrophysics we deal with lifeless, inorganic matter! How to achieve reliability? No jet without abundant jet substance, or rather jet 'plasma', which should be much lighter than its surrounding medium - for confinement purposes - i.e., much smaller in rest mass, and much hotter. This constraint is optimised by relativistic pair plasma, the lightest medium in the Universe; whereby its lightness still increases with the size of its Lorentz factor.

This jet plasma must be supplied continually, throughout the lifetime of a jet engine. We know that our Sun can generate relativistic $\mathrm{e}^{ \pm}$-pairs, at a low rate, even nowadays, in its hot corona. When the Sun was young, born at the inner edge of its (planetary) accretion disk, with a spin period of 3.6 hours (instead of the present 27.3 days), its pair-formation rate has been estimated to have well sufficed to power its Young-Stellar-Object (YSO) stage [Blome \& Kundt, 1988, Kundt, 2005, Kundt \& Marggraf, 2014]. Whereby the necessary magnetic reconnections are thought to result from 'magnetic spanking' of the inner edge of its accretion disk, which serves as a heavy and sharp-edged obstacle. Correspondingly, forming white dwarfs, as well as neutron stars surrounded by accretion disks are expected to be even stronger generators of relativistic pair plasma (than YSOs), in perfect agreement with the observations.

Continual supply of abundant (relativistic) pair plasma is a necessary though not a sufficient condition on a jet engine to function: The freshly generated plasma must be post-accelerated, and funneled into two antipodal channels. We maintain that for the above-listed engines, the funneling of their twin-jets is achieved by buoyancy, in the quasi-spherical gravitational potential of their central rotator, and that the bunching of the charges in momentum space, and their significant postacceleration take place via (i) their just-mentioned buoyancy, further by (ii) their scattering on the ambient photon gas, via (iii) phase-riding on the strong, outgoing low-frequency electromagnetic waves (LFW) of the central rotator - like by the Crab nebula's pulsar [Kulsrud et al, 1972] - and via (iv) profiting from the deLaval-nozzle structure of its ambient medium, as was first proposed by Blandford \& Rees in 1974, see [Kundt \& Krishna, 1980, 2004].

And what is the working pattern of the Central Engines (CEs) of Active Galactic Nuclei (AGN), during their - quite similar-looking - jet formations? As is known since the 1970s, a supermassive black hole (SMBH) cannot anchor a corotating magnetosphere; it can only swallow. No pair formation, no buoyant escape, no LFWs. Fortunately, ever since 1978, I have convinced 
myself (and even a few of my referees) that the CEs of AGN are not SMBHs, rather (nuclear-) Burning Disks (BDs), which act analogously to heavy, magnetised rotators, via their strongly shearing inner-galactic diskal coronas [Kundt 1979, 1990, 1996, 2001, 2005, 2009a,b; 2011a,b,c, Kundt \& Krishna 1980, 2004]. They share the magnetic reconnections, the buoyant escape, the postacceleration by their LFWs, and self-blown deLaval nozzles with the stellar CEs, thus creating quite similar-functioning twin jets.

Apart from their inability to blow jets, Black Holes ought no longer to be considered as potential astrophysical sources, ever since [Pankaj Joshi, 2009, 2013] has reminded us that BHs form a measure-zero subset of the class of all gravitational collapse solutions: Expected during collapse if at all - would be Naked Singularities (NSs); which are, however, not wanted for other reasons.

And even NSs are no longer wanted, by me and by a small group of insiders. Because we all erred, during the 1970s, when we expected (heavy) neutron stars to turn unstable under strong mass accretion, and to crash, (because pressures have weight, and fail eventually to yield support), so that very massive bodies collapse under their own weight, and turn singular. Instead, accreting neutron stars get 'recycled', i.e. are spun up by accretion, due to the huge angular momentum of their added mass, and evolve towards compact disks, i.e. fail to turn singular. No BHs form.

So let us return to our consideration of jet engines. A complete description of their detailed functioning, their dynamics, stabilities, and various morphologies, would take more space than is available here. The reader can find it in the quoted literature. Suffice it to mention that pair formation via magnetic reconnections has been recently assessed by [Dal Pino et al, 2010], that pair annihilations in neutron-star jet sources have been first reported by [Kaiser \& Hannikainen, 2002], that functional regularities in micro-quasars and quasars are described in [Körding et al, 2006, 2008], and that earlier interpretations of similar kinds of the jet phenomenon were given by [Phil Morrison, 1981], and by [Peter Scheuer, 1996]. Finally, if the CEs of the AGN are BDs rather than BHs, the rare, gigantic, supersoft X-ray outbursts reported by [Komossa, 2005] may have to be re-interpreted as caused by innermost stars on perturbed orbits colliding with the heavy innermost disk of their galaxy.

All this said, the reader will still find a short review of above-sketched universal jet model in the next section. At the same time, this review will generalise its earlier versions by allowing for channel-wall material of non-negligible density, impacted by dense surroundings, i.e. by allowing for 'dressed jets'.

\section{My (improved) Semi-Analytical Jet Model}

(i) In order to blow a relativistic twin jet of power L, one needs a heavy generator of $\dot{N}=$ $\mathrm{L} / 2 \gamma \mathrm{m}_{e} \mathrm{c}^{2}=10^{45.7} \mathrm{~s}^{-1} \mathrm{~L}_{44} / \gamma_{4}$ electron-positron pairs per time, of mean Lorentz factor $\gamma \approx 10^{4}$, which we conceive of as a rotating magnetosphere rubbing (shearing) against the inner edge of a surrounding gaseous accretion disk, but the precise mode of magnetic reconnection should not matter, cf. [Kundt 1996, 2001, 2002]. In the case of our present Sun, pair formation at its surface may rather be due to reconnections of excess magnetic flux dragged out of its convection zone by the escaping solar wind, but only at a low rate $\dot{N}$, insufficient for jet formation. 
At the same time, we have learned from pulsars that a rotating magnetosphere of angular velocity $\omega$ emits strong, low-frequency spherical waves of (large) strength parameter $\mathrm{f}$ :

$$
f:=e B / m_{e} c \omega=10^{14.2} B_{3} / \omega_{-4}
$$

for a typical coronal field of strength $B$ measured in $\mathrm{kG}$, with $\mathrm{B}_{3}:=\mathrm{B} / 10^{3} \mathrm{G}, \omega_{-4}:=\omega / 10^{-4} \mathrm{~s}^{-1}$, which post-accelerates charges of either sign to an asymptotic Lorentz factor $\gamma$ of order $\mathrm{f}^{2 / 3}=$ $10^{9.5}\left(\mathrm{~B}_{3} / \omega_{-4}\right)^{2 / 3}$, in the absence of damping [Kulsrud et al, 1972]. Some such damping is expected to occur via scattering on the ambient photon bath, whose main effect will be to narrow the energy distribution of the escaping charges, as is known from laboratory experiments where atomic beams are routinely cooled via scattering on laser light. On the other hand, only $10 \%$ of all AGN have low enough inverse-Compton losses to blow jets, the rest of them are radio quiet, or even radio silent, through exactly such collisional losses on the photon bath of the BLR [Jiang et al, 2007].

Taken together, the newly created pair plasma is antipodally funneled by buoyancy, and strongly boosted by the outgoing low-frequency (LF) waves. Both the LF waves, and the more isotropic High-Frequency (HF) background radiation tend to narrow the energy distribution of the escaping relativistic electrons (of the radio-loud subpopulation) towards a relativistic Maxwellian, whereupon their subsequent feeding into two antipodal jets - by two heavy thermal deLaval nozzles has been shown to further narrow the distribution towards a mono-energetic (delta) flow [Kundt \& Krishna, 2004]. Its $\mathbf{E} \times \mathbf{B}$-drifting rearranges the speeds of the charges until they are uniform across the beam. (Remember that the radio spectrum of Sgr A* is consistent with monoenergetic synchrotron radiation, with $\gamma \gtrsim 10^{4}$ ). In this way, their propagation is loss-free, apart from inverseCompton losses on the background radiations.

(ii) Beyond a deLaval nozzle, the relativistic charges - of bulk Lorentz factor $\gamma \gtrsim 10^{4}$ rearrange their velocities in an orderly manner as $\mathbf{E} \times \mathbf{B}$-drifters, $\beta=\mathbf{E} \times \mathbf{B} / \mathrm{B}^{2}$, whereby in a cylindical section of a naked beam, the toroidal B-fields and radial electric Hall fields imply unique charge- and current-densities $\{\rho, j\}$, smoothly distributed throughout the (naked) beam, whose lowest-order Fourier components read:

$$
\begin{aligned}
E_{s} & =B_{\phi}=C \sin (\pi s / R) / s, B_{z}=\mathrm{const} \\
\rho & =j_{z} / c=(\pi C / R) \cos (\pi s / R) / s
\end{aligned}
$$

in cylindrical coordinates $\{\mathrm{s}, \varphi, \mathrm{z}\}$, with $\mathrm{R}=$ cylinder radius; $\beta_{\varphi}$ turns out to be small, of order $\gamma^{-1}$, unrelated to observed spiral patterns, see Fig.1. Note that such beams have vanishing net charges and currents, and that their charge amplitudes are of order $10^{-10}$ of those of the neutral flows. Fields and charges are in equipartition, and determined by the power $\mathrm{L}$ of a beam and its cross section A via:

$$
n_{e} \gamma m_{e} c^{2} \approx L / A c \approx\left(E^{2}+B^{2}\right) / 8 \pi \approx B^{2} / 4 \pi
$$

for a pair-plasma number density $n_{e}$. Note that these field strengths correspond to (huge) convected electric potentials $\Phi$ given by

$$
\mathrm{e} \Phi \approx \mathrm{e}(\pi \mathrm{L} / \mathrm{c})^{1 / 2}=10^{19.5} \mathrm{eVL}_{44}^{1 / 2},
$$

which guarantee electron energies up into the $\mathrm{PeV}$ range in the ultimate power-law distribution of the charges, once they have been stalled by a heavy obstacle. No stochastic (in situ) acceleration is required anywhere in the beams. 


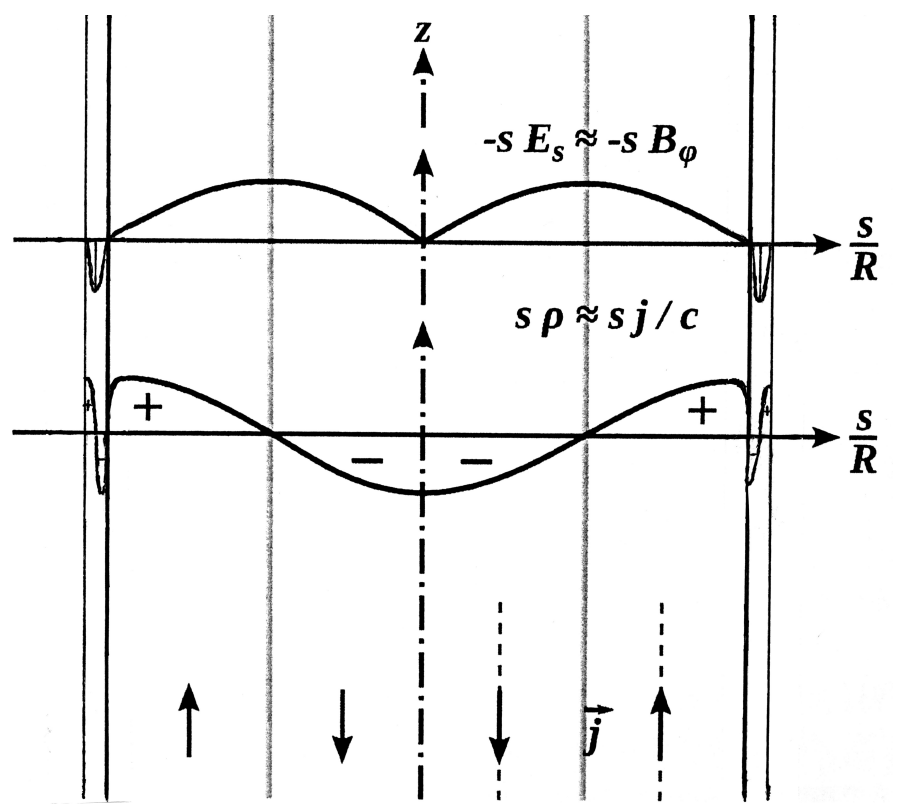

Figure 1: Cross section through (the stable ground mode of) a cylindrical dressed-beam section, showing the radial dependences $(\sim \mathrm{s} / \mathrm{R})$ of $\left\{\rho, \mathrm{j}, \mathrm{E}_{s}, \mathrm{~B}_{\psi}\right\}=\{$ charge density, current density, radial electric, toroidal magnetic field $\}, \mathrm{R}:=$ beam radius. An essential assumption has been that also dressed beams are electrically neutral, and current-free.

Note also that the $\mathbf{E} \times \mathbf{B}$-drifting leptons described by equns. (2.2) and by $\mathrm{c}(\gamma \beta)^{\circ}=\left(\mathrm{e} / \mathrm{m}_{e}\right)(\mathbf{E}+$ $\beta \times \mathbf{B})$ have their dominating losses through inverse Compton collisions on the $2.73 \mathrm{~K}$ background radiation, with a (large) degradation e-folding length $1_{\text {deg }}$ given by

$$
l_{\mathrm{deg}}:=\gamma / \gamma^{\prime}=3 m_{e} c^{2} / 4 \sigma_{\mathrm{T}} \mathrm{u}_{3 \mathrm{~K}} \gamma=\mathrm{Mpc} / \gamma_{6}(1+z)^{4},
$$

which puts only marginal restrictions on the longest observed jet sources.

(iii) What happens when a pair-plasma beam is stalled by an extended, heavy conductor? Such conductive obstacles form naturally, as (part of the) swept-up and heated circumsource matter. Already before any mechanical contact, the approaching, polarized beam plasma will induce mirror charges, and mirror currents in the obstructing plasma, which mediate the transfer of its impact momentum to the 'head material', and more or less reflect the beam particles into the (expanding) 'lobe', or 'cocoon', which is thereby inflated. This momentum transfer, of course, implies a small energy transfer to the (compressed) head material. At the same time, a large fraction of the comoving electromagnetic energy is transferred to the pair plasma, converting its delta-type energy distribution into a broad power-law distribution, and energizing it such that it squeezes the ambient (thermal) circumsource plasma into high-pressure, small-filling-factor filaments. Such explosive compression is known to be Rayleigh-Taylor unstable during its switch-on phase, like water being blown apart by pressurized air. Only later, during the switch-off phase of rapid relaxation, does the lobe develop a smooth, Rayleigh-Taylor stable outer envelope (of expanded cocoon matter), observed as its bounding, shocked outer layer.

Blowing the cocoon consumes one third of the stalled beam-plasma's energy: The pressure 


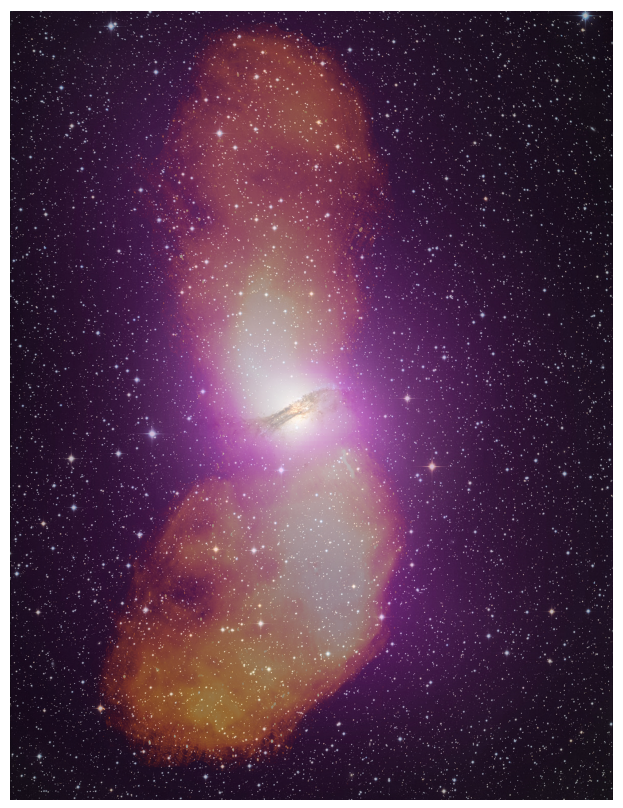

Figure 2: Multifrequency map of our nearest elliptical (radio) galaxy, rather $\gamma$-ray galaxy Cen A, at distance $\mathrm{d}=3 \mathrm{Mpc}$, plotted by NASA. It maps visible colours naturally, radio frequencies in orange, and $\mathrm{MeV}$ energies in purple. Its feeding jets are not resolved on this (largest) $\lesssim \mathrm{Mpc}$ scale.

$p$ of a relativistic gas equals one third of its energy density $u, p=u / 3$. Consequently, in order to compress pre-existing plasma near the distal end of the cocoon into a tiny subvolume, the relativistic gas has to perform work given by $p$ times its volume $V$, which amounts to $1 / 3$ of its original energy $\mathrm{uV}$. This means that the cocoon of a bipolar flow (=: BF) is still a powerful container of relativistic pair plasma, storing two thirds of its energy at injection. No wonder that Cen A, one of the nearest radio galaxies, has been recently mapped at $\mathrm{MeV}$ energies, glowing with more than ten times its radio power; [Teddy Cheung, 2010] has called it a 'gamma-ray galaxy', see Fig.2. And [Pakull et al, 2010] have concluded that $S 26$ in the Sculptor galaxy NGC 7793 was a (10 $5.3 \mathrm{yr}$ old) microquasar of (excessive mechanical) power $\mathrm{L}=10^{40.7} \mathrm{erg} / \mathrm{s}$, from the optical emission of its radio lobes, whereby they omitted a likely filling factor $\mathrm{f}$ of order $10^{-3}$ of the radiating lobe material, corresponding to an f-times lower radiating mass, and an f-times lower inferred mechanical power $\mathrm{f} L=10^{37.7} \mathrm{erg} / \mathrm{s}$ of the microquasar. With such a filling factor included, their source smoothly joins the class of BFs from binary neutron stars, as an older brother of SS 433.

And what about occasional recent reports of TeV sources, or even PeV sources among the members of the jet family? No need for stochastic (in situ) acceleration: When the huge convected potential $\Phi$ found in (2.4) is discharged, at the termination shock of a jet, a comparison with Michel's relativistic Child's Law for a space-charge limited discharge, borrowed from the (assumed) polar-cap discharges of pulsars, yields an expected upper-end (of the power law) Lorentz factor $\gamma_{\infty}$ of order:

$$
\gamma_{\infty} \approx\left(8 \gamma_{\Phi}\right)^{1 / 2} \approx 10^{7.2}\left(\mathrm{~L}_{44}\right)^{1 / 4},
$$

large enough to explain all the recent reports of TeV sources among the BFs as inverse-Compton radiation from the relativistic pair plasma of their jet engines. 
(iv) All our above formulae referred to naked jets, or bare jets, i.e. to jets propagating through (extreme) vacuum. They are symmetrical w.r.t. a sign reversal of their charges, for which electrons and positrons exchange their positions. For all practical purposes, these two subsets of solution look indistinguishable. Or is one of the two subsets excluded in the real world, caused by the asymmetry of protons and electrons in their surrounding medium, which impact on them during jet formation, inside the deLaval nozzle? For the sake of dressing such jets (with ambient matter), let us choose the signs of their excess charges as in Fig.1 above; they should matter. Impacting ions from their CSM will then be electrostatically repelled from it, and at the same time dragged along by the relativistic $\mathrm{e}^{ \pm}$pairs from the unperturbed flow - at roughly comparable particle momenta for sufficiently slow ions - such that they end up co-moving alongside at subrelativistic speeds with the bare jet, in a thin $\left(\gtrsim 10^{-3} \mathrm{R}\right)$ boundary layer indicated in Fig.1, whose constituents are (intruded) ions plus a certain density of positrons, their sum quasi neutralised by a comparable number of (formerly ambient) electrons.

This dressed-beam model has been based on the assumptions of electric neutrality of jets and of a vanishing net current along them. Its best known application is the neutron-star binary SS 433, with its fast-moving ions at both X-ray and optical photon energies; but also most of the HighVelocity Clouds (HVCs) and Intermediate-Velocity Clouds (IVCs) of the Milky Way's halo may owe their existence to a more powerful past of our Galactic AGN [Kundt, 1997]. As concerns the SS 433 system, note that none of its published evaluations are satisfactory - they mutually differ in model, distance, mass, power, orientations, rotation senses, and (the quality of) correlations and arrive at different speeds $\mathrm{v}=\mathrm{c} \beta$ with $\beta=1 / 8 \cos (\mathrm{i}-\Theta)=\{1 / 8,1 / 4\}$ for $\mathrm{i}-\Theta=\left\{0,60^{\circ}\right\}$. We (presently) prefer $\beta \lesssim 1 / 4$.

(v) The heads of young jets move fast enough to penetrate supersonically into their surroundings, whilst those of older copies tend to fall short of this critical speed, and expand only subsonically. As is well known, the morphologies of these two source classes differ strongly. Jean Eilek [2002] has termed them of type $A$ and type $B$ respectively, in slight (though important) revision of the earlier Fanaroff \& Riley classification of double radio sources.

\section{Summary of the Model}

In short, the key properties of my updated jet model are as follows: No jets, or bipolar flows, without a rotating magnet, whose magnetic reconnections supply the necessary (relativistic) pair plasma, and whose outgoing low-frequency waves provide the necessary post-acceleration of the leptonic charges. In this process, inverse-Compton losses on the ambient photon bath must not be excessive, otherwise we deal with radio-silent QSOs (without jets). Supersonic E x B-drifting jets emerge at two opposite outlets of the (central) BLR, via naturally forming deLaval nozzles, whereby equipartition electromagnetic fields convect half of the escaping power through their selframmed jet channels. They serve as comoving batteries wherever the beams get tapped by obstacles, most notably at their downstream shocks (heads). At their heads, the jets blow the lobes, which are observed as low-density relativistic balloons with thermal inclusions of small filling factor, and with spectra which extend up in energy to the VHE $\gamma$-ray regime.

But already during their formation, in passing from subsonic to supersonic propagation, inside their deLaval nozzles, the forming jets are strong $\gamma$-ray emitters, with Lorentz factors reaching 
up to $\gamma \lesssim 10^{6}$. And embedding gas and/or plasma is dragged along by them in the form of thin, marginally subrelativistc channel-wall bandages, best exemplified by the Galactic X-ray binary SS 433. Weakest among all jet sources are those blown by brown dwarfs.

\section{Acknowledgements}

My cordial thanks for the manuscript, including its contents, go to Ole Marggraf.

\section{References}

[1] Blome, H.-J., Kundt, W.: 1988, Leptonic Jets from Young Stellar Objects?, Astrophys. \& Space Sci. 148, 343-361.

[2] Dal Pino, E.M. de G., Piovezan, P.P., Kadowaki, L.H.S.: 2010, Astron. Astrophys. 518; arXiv: $1005.3067 \mathrm{v} 1$.

[3] Jiang, L., Fan, X., Ivecic, Z., Richards, G.T., Schneider, D.P., Strauss, M.A., Kelly, B.C.: 2007, Astrophys. J. 656, 680-690.

[4] Joshi, P.S.: 2009, Naked Singularities, Scientific American, February, 22-33; literally reprinted in May 2013.

[5] Kaiser, C.R., Hannikainen, D.C.: 2002, MNRAS 330, 225-231.

[6] Körding, E.G., Jester, S., Fender, R.: 2006, MNRAS 372, 1366-1378.

[7] Körding, E.G., Jester, S., Fender, R.: 2008, MNRAS 383, 277-288.

[8] Komossa, S.: 2005, in Growing Black Holes, ESO Symposia, Springer, 159-163.

[9] Kulsrud, R.M., Ostriker, J.P., Gunn, J.E.: 1972, Phys. Rev. Lett. 28, 636-639.

[10] Kundt, W.: 1979, A Model for Galactic Centers, Astrophys. \& Space Science 62, 335-345.

[11] Kundt, W.: 1990, The Galactic Centre, Astrophys. \& Space Sci. 172, 109-134.

[12] Kundt, W.: 1996, in Jets from Stars and Galactic Nuclei, Lecture Notes in Physics 471, Springer, W. Kundt (ed.), 140-144.

[13] Kundt, W.: 1997, Structure of the Galactic Halo and Disk, in: The Physics of Galactic Halos, eds. H. Lesch, R.-J. Dettmar, U. Mebold, \& R. Schlickeiser, Akademie Verlag, 255-259.

[14] Kundt, W.: 2001, Jet Formation and Dynamics: Comparison of Quasars and Microquasars, in MICROQUASARS, Proceedings of the Third Miroquasar Workshop, A.J. Castro-Tirado, J. Greiner \& J.M. Paredes (eds.), Kluwer, 273-277.

[15] Kundt, W.: 2002: Radio Galaxies powered by Burning Disks, in: Life Cycles of Radio Galaxies, eds. J.A. Biretta, A.M. Koekemoer, E.S. Perlman, \& C.O. O’Dea, New Astronomy Reviews 46, 2-7, pp. 257-261.

[16] Kundt, W.: 2005, Astrophysics, A New Approach, Springer, Chapter 11.

[17] Kundt, W.: 2009a, Critical Thoughts on Cosmology, in XIIIth Brazilian School of Cosmology \& Gravitation, AIP Conf. Proc. 1132, M. Novello \& S.E.P. Bergliaffa (eds.), Melville, N.Y., 288-302.

[18] Kundt, W.: 2009b, Jürgen Ehlers and the fate of the Black-Hole Spacetimes, in General Relativity and Gravitation 41, Nr. 9, 1967-1980. 
[19] Kundt, W.: 2011a, The Astrophysical Jets, in Jets at All Scales, IAU Symposium No.275 in Buenos Aires, eds. Gustav Romero, Rashid Sunyaev \& Tomaso Belloni, Cambridge Univ. Press, pp. 92-93.

[20] Kundt, W.: 2011b, The Astrophysical Jets - after 30 years of deliberation, in Frontier Objects in Astrophysics and Particle Physics, Conference Proceedings Vol. 103, Vulcano Workshop 2010, eds. F. Giovannelli \& G. Mannocchi, Bologna, pp.389-404.

[21] Kundt, W.: 2011c, Black Holes cannot blow Jets, in (COSMOLOGY and GRAVITATION), XIV ${ }^{\text {th }}$ Brazilian School of Cosmology and Gravitation, Mangaratiba, eds. Mario Novello \& Santiago Bergliaffa, Cambridge Scientific Publishers, pp. 109-118.

[22] Kundt, W.: 2015, A Uniform Description of All the Astrophysical Jets, Mondello 2014, PoS(FRAPWS2014)025, 1-9(2015).

[23] Kundt, W., Gopal-Krishna: 1980, Nature 288, 149-150.

[24] Kundt, W., Krishna, G.: 2004, The Physics of E $\times$ B-drifting Jets, J. Astrophys. Astr. 25, 115-127.

[25] Kundt, W., Marggraf, O.: 2014, Physikalische Mythen auf dem Prüfstand, Springer, Kapitel 7.

[26] Morrison, P.: 1981, unpublished evening lecture at Socorro, during IAU Symposium 97 at Albuquerque. (on Extragalactic Radio Sources formed from matter and anti-matter).

[27] Scheuer, P.A.G.: 1996, in Jets from Stars and Galactic Nuclei, Lecture Notes in Physics 471, Springer, W. Kundt (ed.), 35-40. 